\title{
Quantum probabilities as Bayesian probabilities
}

\author{
Carlton M. Caves, ${ }^{(1)}$ Christopher A. Fuchs, ${ }^{(1)}$ and Rüdiger Schack ${ }^{(2)}$ \\ ${ }^{1}$ Bell Labs, Lucent Technologies, 600-700 Mountain Avenue, Murray Hill, New Jersey 07974, USA \\ ${ }^{2}$ Department of Mathematics, Royal Holloway, University of London, Egham, Surrey TW20 0EX, United Kingdom
}

(23 September 2001)

In the Bayesian approach to probability theory, probability quantifies a degree of belief for a single trial, without any a priori connection to limiting frequencies. In this paper we show that, despite being prescribed by a fundamental law, probabilities for individual quantum systems can be understood within the Bayesian approach. We argue that the distinction between classical and quantum probabilities lies not in their definition, but in the nature of the information they encode. In the classical world, maximal information about a physical system is complete in the sense of providing definite answers for all possible questions that can be asked of the system. In the quantum world, maximal information is not complete and cannot be completed. Using this distinction, we show that any Bayesian probability assignment in quantum mechanics must have the form of the quantum probability rule, that maximal information about a quantum system leads to a unique quantum-state assignment, and that quantum theory provides a stronger connection between probability and measured frequency than can be justified classically. Finally we give a Bayesian formulation of quantum-state tomography.

\section{INTRODUCTION}

There are excellent reasons for interpreting quantum states as states of knowledge [1]. A classic argument goes back to Einstein [2]. Take two spatially separated systems $A$ and $B$ prepared in some entangled quantum state $\left|\psi^{A B}\right\rangle$. By performing the measurement of one or another of two observables on system $A$ alone, one can immediately write down a new state for system $B$ - either a state drawn from a set $\left\{\left|\phi_{i}^{B}\right\rangle\right\}$ or a set $\left\{\left|\eta_{i}^{B}\right\rangle\right\}$, depending upon which observable is measured. Since this holds no matter how far apart the two systems are, Einstein concluded that quantum states cannot be "real states of affairs." For whatever the real, objective state of affairs at $B$ is, it should not depend upon the measurements made at $A$. If one accepts this conclusion, one is forced to admit that the new state (either a $\left|\phi_{i}^{B}\right\rangle$ or a $\left|\eta_{i}^{B}\right\rangle$ ) represents partial knowledge about system $B$. In making a measurement on $A$, one learns something about $B$; the state itself cannot be construed to be more than a reflection of the new knowledge.

The physical basis of Einstein's argument has recently become amenable to experimental test. Zbinden et al. 33 have reported an experiment with entangled photons in which the detectors at $A$ and $B$ are in relative motion.
The experimental data rule out a certain class of realistic collapse models, i.e., models in which the real state of affairs at $B$ changes as a result of the measurement at $A$. They also put a lower bound of $10^{7}$ times the speed of light on the speed of any hypothetical quantum influence of the measurement at $A$ on the real state of affairs at $B$.

We accept the conclusion of Einstein's argument and start from the premise that "quantum states are states of knowledge." An immediate consequence of this premise is that all the probabilities derived from a quantum state, even a pure quantum state, depend on a state of knowledge; they are subjective or Bayesian probabilities. We outline in this paper a general framework for interpreting all quantum probabilities as subjective.

If two scientists have different states of knowledge about a system, they will assign different quantum states, and hence they will assign different probabilities to the outcomes of some measurements. This situation is commonly encountered in quantum cryptographic protocols [4], where the different players, possibly including an eavesdropper, have different information about the quantum systems they are handling. In a Bayesian framework, the probabilities assigned by the different players are all treated on an equal footing; they are all equally valid. Subjective probability has, therefore, no a priori connection to measured frequencies and applies naturally to single quantum systems.

The Bayesian approach has been very successful in statistics [5 [7], observational astronomy 8], artificial intelligence 9], and classical statistical mechanics [10]. It seems to be the general opinion, however, that the Bayesian interpretation is not suitable for quantummechanical probabilities. The probabilities that come from a pure state are intrinsic and unavoidable. How can they not be objective properties when they are prescribed by physical law? How can Bayesian quantum state assignments be anything but arbitrary? Hasn't the tight connection between probability and measured frequencies been verified in countless experiments? What is an experimenter doing in quantum-state tomography 11, 12 if not determining the unknown objective quantum state of a system?

In this paper we give answers to these questions. These answers turn out to be simple and straightforward. After a brief introduction to Bayesian probability theory, we use Gleason's theorem 13] to show that any subjective quantum probability assignment must have the form of the standard quantum probability rule. We then use a 
version of the so-called Dutch-book argument [14,15, to show that if a scientist has maximal information about a quantum system, he must assign a unique pure state. Our next step is to show that in the case of maximal information, there is a conceptually simple connection between (subjective) probability and (measured) frequency, which is tighter than can be justified classically. Finally, we consider quantum-state tomography, where an experimenter is said to be determining the "unknown quantum state" of a system from the results of repeated measurements on many copies of the system. An "unknown quantum state" is an oxymoron if quantum states are states of knowledge, and we show how it can be eliminated from the description of tomography by using a quantum version of the de Finetti representation theorem for exchangeable sequences [16,17]. We conclude with a brief summary and an outlook.

\section{BAYESIAN PROBABILITY AND THE DUTCH BOOK}

Bayesian probabilities are degrees of belief or uncertainty [6], which are given an operational definition in decision theory [5], i.e., the theory of how to decide in the face of uncertainty. The Bayesian approach captures naturally the notion that probabilities can change when new information is obtained. The fundamental Bayesian probability assignment is to a single system or a single realization of an experiment. Bayesian probabilities are defined without any reference to the limiting frequency of outcomes in repeated experiments. Bayesian probability theory does allow one to make (probabilistic) predictions of frequencies, and frequencies in past experiments provide valuable information for updating the probabilities assigned to future trials. Despite this connection, probabilities and frequencies are strictly separate concepts.

The simplest operational definition of Bayesian probabilities is in terms of consistent betting behavior, which is decision theory in a nutshell. Consider a bookie who offers a bet on the occurrence of outcome $E$ in some situation. The bettor pays in an amount $p x$ - the stake - up front. The bookie pays out an amount $x$ - the payoff - if $E$ occurs and nothing otherwise. Conventionally this is said to be a bet at odds of $(1-p) / p$ to 1 . For the bettor to assign a probability $p$ to outcome $E$ means that he is willing to accept a bet at these odds with an arbitrary payoff $x$ determined by the bookie. The payoff can be positive or negative, meaning that the bettor is willing to accept either side of the bet. We call a probability assignment to the outcomes of a betting situation inconsistent if it forces the bettor to accept bets in which he incurs a sure loss; i.e., he loses for every possible outcome. A probability assignment will be called consistent if it is not inconsistent in this sense.

Remarkably, consistency alone implies that the bettor must obey the standard probability rules in his probability assignment: (i) $p \geq 0$, (ii) $p(A \vee B)=p(A)+p(B)$ if $A$ and $B$ are mutually exclusive, (iii) $p(A \wedge B)=$ $p(A \mid B) p(B)$, and (iv) $p(A)=1$ if $A$ is certain. Any probability assignment that violates one of these rules can be shown to be inconsistent in the above sense. This is the so-called Dutch-book argument [14]15]. We stress that it does not invoke expectation values or averages in repeated bets; the bettor who violates the probability rules suffers a sure loss in a single instance of the betting situation.

For instance, to show that $p(A \vee B)=p(A)+p(B)$ if $A$ and $B$ are mutually exclusive, assume that the bettor assigns probabilities $p_{A}, p_{B}$, and $p_{C}$ to the three outcomes $A, B$, and $C=A \vee B$. This means he will accept the following three bets: a bet on $A$ with payoff $x_{A}$, which means the stake is $p_{A} x_{A}$; a bet on $B$ with payoff $x_{B}$ and thus with stake $p_{B} x_{B}$; and a bet on $C$ with payoff $x_{C}$ and thus with stake $p_{C} x_{C}$. The net amount the bettor receives is

$R= \begin{cases}x_{A}\left(1-p_{A}\right)-x_{B} p_{B}+x_{C}\left(1-p_{C}\right) & \text { if } A \wedge \neg B \\ -x_{A} p_{A}+x_{B}\left(1-p_{B}\right)+x_{C}\left(1-p_{C}\right) & \text { if } \neg A \wedge B \\ -x_{A} p_{A}-x_{B} p_{B}-x_{C} p_{C} & \text { if } \neg A \wedge \neg B\end{cases}$

The outcome $A \wedge B$ does not occur since $A$ and $B$ are mutually exclusive. The bookie can choose values $x_{A}$, $x_{B}$, and $x_{C}$ that lead to $R<0$ in all three cases unless

$$
0=\operatorname{det}\left(\begin{array}{ccc}
1-p_{A} & -p_{B} & 1-p_{C} \\
-p_{A} & 1-p_{B} & 1-p_{C} \\
-p_{A} & -p_{B} & -p_{C}
\end{array}\right)=p_{A}+p_{B}-p_{C} .
$$

The probability assignment is thus inconsistent unless $p(A \vee B)=p_{C}=p_{A}+p_{B}$.

In our experience physicists find it difficult first to accept and then to embrace the notion that subjective probabilities receive their only operational significance from decision theory, the simplest example of which is the Dutch-book argument in which probabilities are defined to be betting odds. In the Dutch-book approach the structure of probability theory follows solely from the requirement of consistent betting behavior. There is no other input to the theory. For example, normalization of the probabilities for exclusive and exhaustive alternatives is not an independent assumption, so obvious that it needs no justification. Instead normalization follows from probability rules (ii) and (iv) above and thus receives its sole justification from the requirement of consistent betting behavior.

The only case in which consistency alone leads to a particular numerical probability is the case of certainty, or maximal information. If the bettor is certain that the outcome $E$ will occur, the probability assignment $p<1$ means he is willing to take the side of the bookie in a bet on $E$, receiving an amount $p x$ up front and paying out $x$ if $E$ occurs, leading to a certain loss of $x(1-p)>0$. Consistency thus requires that the bettor assign probability 
$p=1$. More generally, consistency requires a particular probability assignment only in the case of maximal information, which classically always means $p=1$ or 0 .

The quantum situation is radically different, since in quantum theory maximal information is not complete [18]. This notwithstanding, we show that consistency still requires particular probability assignments in the case of maximal information and, what is more, that these probabilities are numerically equal to expected limiting frequencies. The keys to these results are Gleason's theorem and a quantum variant of the Dutch-book argument of the previous paragraph.

\section{GLEASON'S THEOREM AND THE QUANTUM PROBABILITY RULE}

In order to derive the quantum probability rule, we make the following assumptions about a quantum system that is described by a $D$-dimensional Hilbert space:

(i) Each set of orthogonal one-dimensional projectors, $\hat{\Pi}_{k}=\left|\psi_{k}\right\rangle\left\langle\psi_{k}\right|, k=1, \ldots, D$ (the vectors $\left|\psi_{k}\right\rangle$ make up an orthonormal basis), corresponds to the complete set of mutually exclusive outcomes of some measurement, i.e., answers to some question that can be posed to the system. Throughout this paper, what we mean by a "quantum question" is a measurement described by such a complete set of orthogonal one-dimensional projectors.

(ii) The probabilities assigned to the outcomes are consistent in the Dutch-book sense given above.

(iii) The probability assignment is noncontextual [20]; i.e., the probability for obtaining the outcome corresponding to a projector $\hat{\Pi}$ depends only on $\hat{\Pi}$ itself, not on the other vectors in the orthogonal set defining a particular measurement. As a consequence, it can be denoted $p(\hat{\Pi})$.

Condition (ii) implies that, for each set of orthogonal one-dimensional projectors,

$$
\sum_{k=1}^{D} p\left(\hat{\Pi}_{k}\right)=1
$$

Of course, this is simply the normalization condition, but in the Bayesian view, normalization is enforced only by the requirement of consistent betting behavior. Except in the special case of a two-dimensional Hilbert space, condition (iii) then implies that there exists a density operator $\hat{\rho}$ such that for every projector $\hat{\Pi}=|\psi\rangle\langle\psi|$,

$$
p(\hat{\Pi})=\operatorname{tr}(\hat{\rho} \hat{\Pi})=\langle\psi|\hat{\rho}| \psi\rangle .
$$

This is Gleason's theorem [13]. It means that, under the assumptions of (i) the Hilbert-space structure of quantum questions, (ii) Dutch-book consistency, and (iii) probabilities reflecting the Hilbert-space structure, any subjective probability assignment must have the form (4), which is the standard quantum rule for probabilities. Hence Bayesian "degrees of belief" are restricted by the laws of nature, and any subjective state of knowledge about a quantum system can be summarized in a density operator $\hat{\rho}$. Since one of the chief challenges of Bayesianism is the search for methods to translate information into probability assignments, Gleason's theorem can be regarded as the greatest triumph of Bayesian reasoning.

\section{MAXIMAL INFORMATION AND UNIQUE STATE ASSIGNMENT}

Our concern now is to show that if a scientist has maximal information about a quantum system, Dutchbook consistency forces him to assign a unique pure state. Maximal information in the classical case means knowing the outcome of all questions with certainty. Gleason's theorem forbids such all-encompassing certainty in quantum theory. Maximal information in quantum theory instead corresponds to knowing the answer to a maximal number of questions (i.e., measurements described by one-dimensional orthogonal projectors). Suppose then that a scientist is certain about the outcome of all questions that share one particular projector $\hat{\Pi}=|\psi\rangle\langle\psi|$. The scientist is certain that the outcome corresponding to this projector will occur in response to any of these questions, so Dutch-book consistency requires that its probability be $p=1$. Now let $\hat{\rho}$ be the state assigned to the system. In the language of Gleason's theorem, we have $\langle\psi|\hat{\rho}| \psi\rangle=1$. This implies that $\hat{\rho}=\hat{\Pi}=|\psi\rangle\langle\psi|$. Gleason's theorem further implies that the scientist cannot be certain about the outcome of any other questions, so this is the case where he has maximal information. Maximal information thus leads to the assignment of a unique pure state.

Given the assumptions of Gleason's theorem, if a scientist has maximal information, any state assignment that is different from the unique pure state derived in the last paragraph is inconsistent in the Dutch-book sense; i.e., it leads to a sure loss for a bet on the outcome of a measurement on a single system that includes the unique pure state among the outcomes. The Hilbert-space structure of quantum questions plus noncontextuality alone puts this tight constraint on probability assignments.

We emphasize that the uniqueness of the quantum state assignment holds even though no measurement allows an experimenter to decide with certainty between two nonorthogonal pure-state assignments. Though maximal information leads to a unique pure state, the state assignment cannot be verified by addressing questions to the system. Finding out the state assignment requires consulting the assigner or the records he leaves behind. This property is another reason for regarding quantum states as states of knowledge. 
In both the classical and the quantum case, consistency enforces a particular probability assignment if and only if there is maximal information. In the classical case, maximal information corresponds to certainty, i.e., the trivial probability assignment 1 or 0 , so classically maximal information is complete. In quantum mechanics, maximal information leads to a unique pure state assignment $|\psi\rangle\langle\psi|$, which is equivalent to prescribing (generally nontrivial) probabilities for all possible measurements. In quantum mechanics, maximal information is not complete and cannot be completed.

\section{SUBJECTIVE PROBABILITY AND MEASURED FREQUENCY}

Up to this point, we have not mentioned repeated experiments or long-run frequencies. Both the Dutch-book argument and Gleason's theorem are formulated for single systems. There is no justification, at this point, for identifying the probabilities derived from Gleason's theorem with limiting frequencies. To make the connection between the above results and repeated measurements, an additional assumption is needed, namely that the Hilbert space of $N$ copies of a quantum system is given by the $N$-fold tensor product of the single-system Hilbert space. In doing so, we are assuming that the $N$ copies of the quantum system are labeled by some additional degree of freedom that renders irrelevant the symmetries required for identical particles.

Now assume that a scientist has maximal information about $N$ copies of a quantum system, specifically the same maximal information about each system. Applying Gleason's theorem and the Dutch-book argument of Sec. [V] to the tensor-product Hilbert space leads to a unique pure product-state assignment $\hat{\rho}^{(N)}=\hat{\Pi} \otimes \cdots \otimes \hat{\Pi}$, where $\hat{\Pi}=|\psi\rangle\langle\psi|$. Suppose that repeated measurements are performed using the single-system projectors $\hat{\Pi}_{k}=\left|\psi_{k}\right\rangle\left\langle\psi_{k}\right|, k=1, \ldots, D$. The probability of obtaining the sequence of outcomes $k_{1}, \ldots, k_{N}$ is given by

$p\left(k_{1}, \ldots, k_{N}\right)=\operatorname{tr}\left(\hat{\rho}^{(N)} \hat{\Pi}_{k_{1}} \otimes \cdots \otimes \hat{\Pi}_{k_{N}}\right)=p_{k_{1}} \cdots p_{k_{N}}$

where

$$
p_{k}=\operatorname{tr}\left(\hat{\Pi} \hat{\Pi}_{k}\right)=\left|\left\langle\psi_{k} \mid \psi\right\rangle\right|^{2}
$$

This means that the outcomes of repeated measurements are independent and identically distributed (i.i.d.). The probability for outcome $k$ to occur $n_{k}$ times, where $k=$ $1, \ldots, D$ and $\sum_{k} n_{k}=N$, is given by the multinomial distribution,

$$
p\left(n_{1}, \ldots, n_{D}\right)=\frac{N !}{n_{1} ! \cdots n_{D} !} p_{1}^{n_{1}} \cdots p_{D}^{n_{D}},
$$

which peaks for large $N$ at $n_{k} \simeq N p_{k}, k=1, \ldots, D$. The probability of observing frequencies $n_{k} / N$ close to $p_{k}$ converges to 1 as $N$ tends to infinity.

In the classical case an i.i.d. assignment is often the starting point of a probabilistic argument. Yet in Bayesian probability theory, an i.i.d. can never be strictly justified except in the case of maximal information, which in the classical case implies certainty and hence trivial probabilities. The reason is that the only way to be sure all the trials are identical in the classical case is to know everything about them, which implies that the results of all trials can be predicted with certainty 19]. In contrast, to ensure that all systems are the same in quantum mechanics, it is sufficient to have the maximal, but incomplete information that leads to a unique pure state. Thus the quantum i.i.d. assignment (7) is a consequence of Dutch-book consistency and the Hilbert-space structure of quantum mechanics.

To summarize, in quantum mechanics maximal information leads to nontrivial i.i.d. assignments. Maximal information means that the pure product-state assignment is the unique consistent state assignment. From the pure product-state assignment comes the i.i.d. for the outcomes of any repeated measurement. Together with elementary combinatorics, this gives the strict connection between probabilities and frequencies displayed in the laws of large numbers. In this sense, the equality between probability and limiting frequency holds only in quantum mechanics.

\section{UNKNOWN QUANTUM STATES AND THE QUANTUM DE FINETTI REPRESENTATION}

An important practical use of repeated measurements on many copies of a quantum system is in quantum-state tomography [11,12. The data gathered from the measurements is said to determine the "unknown quantum state" of the system. But what can an unknown quantum state mean? If a quantum state is a state of knowledge, then it must be known by somebody. If the Bayesian interpretation of quantum probabilities is to be taken seriously, there must be a way to eliminate the "unknown quantum state" from the description.

The key to this excision is to identify the salient feature of the state of knowledge that applies when an experimenter performs quantum-state tomography. That salient feature is that the experimenter can contemplate examining an arbitrarily large number of systems, all of which are equivalent from his perspective. This means (i) that the density operator $\hat{\rho}^{(N)}$ for $N$ systems should be symmetric, i.e., invariant under all permutations of the $N$ systems, and (ii) that this symmetry should hold for all values of $N$, with the consistency requirement that $\hat{\rho}^{(N)}$ arises from tracing out one of the systems in $\hat{\rho}^{(N+1)}$. A sequence of density operators that satisfies these two properties is said to be exchangeable, by analogy with de 
Finetti's definition [14] of exchangeable multi-trial probabilities.

The quantum de Finetti representation theorem [16, 17 establishes that for any exchangeable sequence of density operators, $\hat{\rho}^{(N)}$ can be written uniquely in the form

$$
\hat{\rho}^{(N)}=\int d \rho p(\rho) \rho \otimes \cdots \otimes \rho,
$$

where the tensor product includes $N$ terms, the integral runs over the space of density operators, and the "generating function" $p(\rho)$ can be thought of as a normalized "probability density on density operators." An exchangeable density operator captures what an experimenter knows about the systems he intends to examine. It is a primary quantum-state assignment for multiple copies, with no mention of unknown quantum states. The content of the quantum de Finetti representation is that the exchangeable state assignment can nevertheless be thought of in terms of unknown density operators; ignorance of which density operator is described by the generating function.

Exchangeability permits us to describe what is going on in quantum-state tomography. Suppose two scientists make different exchangeable state assignments and then jointly collect data from repeated measurements. Suppose further that the measurements are "tomographically complete"; i.e., the measurement probabilities for any density operator are sufficient to determine that density operator. The two scientists can use the data $D$ from an initial set of measurements to update their state assignments for further systems. In the limit of a large number of initial measurements, they will come to agreement on a particular product state $\hat{\rho}_{D} \otimes \hat{\rho}_{D} \otimes \cdots$ for further systems, where $\hat{\rho}_{D}$ is determined by the data. This is what quantum-state tomography is all about. The updating can be cast as an application of Bayes's rule to updating the generating function in light of the data [21]. The only requirement for "coming to agreement" is that both scientists should have allowed for the possibility of $\hat{\rho}_{D}$ by giving it nonzero support in their initial generating functions.

\section{SUMMARY AND OUTLOOK}

We promised simple answers, and it's hard to imagine simpler ones. The physical law that prescribes quantum probabilities is indeed fundamental, but the reason is that it is a fundamental rule of inference - a law of thought - for Bayesian probabilities. It follows from requiring Dutch-book consistency for probability assignments that are faithful to the Hilbert-space structure of quantum questions. These same desiderata require a particular pure-state assignment when a scientist has maximal information, and because maximal information is not complete, they give a strict connection between observed frequencies and pure-state quantum probabilities. The notion of an "unknown quantum state," irreconcilable with the idea of quantum states as states of knowledge, can be banished from quantum-state tomography using the quantum de Finetti representation.

Quantum information science 22] is an emerging field that uses quantum states to escape the constraints imposed on information processing in a realistic/deterministic world. The rewards in quantum information science are great: teleportation of quantum states, distribution of secret keys for encoding messages securely, and computations done more efficiently on a quantum computer than on any classical machine. The key to these rewards is that a quantum world is less constrained than a classical one. As quantum information science harnesses the greater range of possibilities available in the quantum world, we believe it is imperative to understand and elucidate the fundamental principles underlying quantum mechanics. In this paper we show how to interpret quantum states consistently as states of knowledge, reflecting what we know about a quantum system. This is just one step in a broader program to try to disentangle the subjective and objective aspects of the quantum world 24]. We leave the last word to Edwin T. Jaynes [23], who inspired us to pursue the Bayesian view:

Today we are beginning to realize how much of all physical science is really only information, organized in a particular way. But we are far from unravelling the knotty question: "To what extent does this information reside in us, and to what extent is it a property of Nature?" .. O Our present QM formalism is a peculiar mixture describing in part laws of Nature, in part incomplete human information about Nature - all scrambled up together by Bohr into an omelette that nobody has seen how to unscramble. Yet we think the unscrambling is a prerequisite for any further advance in basic physical theory ... .

\section{ACKNOWLEDGMENTS}

CMC was supported in part by U.S. Office of Naval Research Grant No. N00014-93-1-0116.

* Permanent address: Department of Physics and Astronomy, University of New Mexico, Albuquerque, New Mexico 87131-1156, USA

[1] C. A. Fuchs and A. Peres, "Quantum Theory Needs No 'Interpretation'," Phys. Today 53(3), 70 (2000); "Quantum Theory - Interpretation, Formulation, Inspiration: Fuchs and Peres Reply," 53(9), 14 (2000). 
[2] A. Einstein, "Remarks concerning the essays brought together in this co-operative volume," in Albert Einstein: Philosopher-Scientist, 3rd ed., edited by P. A. Schilpp (Open Court, La Salle, IL, 1970), p. 665. Related ideas lie at the core of A. Einstein, B. Podolsky, and N. Rosen, "Can quantum-mechanical description of physical reality be considered complete?" Phys. Rev. 47, 777 (1935).

[3] H. Zbinden, J. Brendel, N. Gisin, and W. Tittel, "Experimental test of nonlocal quantum correlation in relativistic configurations," Phys. Rev. A 63, 022111 (2001).

[4] N. Gisin, G. Ribordy, W. Tittel, and H. Zbinden, "Quantum cryptography," Rev. Mod. Phys., to be published, quant-ph/0101098.

[5] L. J. Savage, The Foundations of Statistics (Dover, New York, 1972).

[6] J. M. Bernardo and A. F. M. Smith, Bayesian Theory (Wiley, Chichester, 1994).

[7] D. Malakoff, "Bayes offers a 'new' way to make sense of numbers," Science 286, 1460 (1999).

[8] T. J. Loredo, "The promise of Bayesian inference for astrophysics," In Statistical Challenges in Modern Astronomy, edited by E. D. Feigelson and G. J. Babu (SpringerVerlag, New York, 1992), p. 275.

[9] Microsoft Research Division's Decision Theory \&6 Adaptive Systems Group homepage: http://www.research.microsoft.com/dtas/.

[10] E. T. Jaynes, Papers on Probability, Statistics and Statistical Physics (Kluwer, Dordrecht, 1983), edited by R. D. Rosenkrantz.

[11] U. Leonhardt and H. Paul, "Measuring the quantum state of light," Prog. Quant. Electron. 19, 89 (1995).

[12] V. Bužek, G. Drobný, R. Derka, G. Adam, and H. Wiedemann, "Quantum state reconstruction from incomplete data," Chaos, Solitons and Fractals 10, 981 (1999).

[13] A. M. Gleason, "Measures on the closed subspaces of a Hilbert space," J. Math. Mech. 6, 885 (1957).

[14] B. de Finetti, Theory of Probability (Wiley, New York, 1990).

[15] J. Earman, Bayes or Bust? A Critical Examination of Bayesian Confirmation Theory (MIT Press, Cambridge, MA, 1992).

[16] R. L. Hudson, and G. R. Moody, "Locally normal symmetric states and an analogue of de Finetti's theorem," Z. Wahrschein. verw. Geb. 33, 343 (1976).

[17] C. M. Caves, C. A. Fuchs, and R. Schack, "Unknown quantum states: The quantum de Finetti representation," submitted to Am. J. Phys., quant-ph/0104088.

[18] C. M. Caves and C. A. Fuchs, "Quantum information: How much information in a state vector?" in The Dilemma of Einstein, Podolsky and Rosen - 60 Years Later, edited by A. Mann and M. Revzen, Ann. Israel Phys. Soc. 12, 226 (1996).

[19] E. T. Jaynes, Probability Theory: The Logic of Science, unpublished, available at http://bayes.wustl.edu.

[20] H. Barnum, C. M. Caves, J. Finkelstein, C. A. Fuchs, and R. Schack, "Quantum probability from decision theory?" Proc. Roy. Soc. London A 456, 1175 (2000).

[21] R. Schack, T. A. Brun, and C. M. Caves, "Quantum Bayes rule," Phys. Rev. A 64, 014305 (2001).

[22] C. H. Bennett and D. P. DiVincenzo, "Quantum infor- mation and computation," Nature 404, 247 (2000).

[23] E. T. Jaynes, "Probability in quantum theory," in Complexity, Entropy and the Physics of Information, edited by W. H. Zurek (Addison-Wesley, Redwood City, CA, 1990), p. 381.

[24] C. A. Fuchs, "Quantum Foundations in the Light of Quantum Information," to appear in Proceedings of the NATO Advanced Research Workshop on Decoherence and its Implications in Quantum Computation and Information Transfer, edited by A. Gonis (Plenum, New York, 2001), quant-ph/0106166. 